\title{
Multimodal Intervention Approach Reduces Catheter-associated Urinary Tract Infections in a Rural Tertiary Care Center
}

Bindu R. Potugari, MD; Peter E. Umukoro MD, MPH, ScD; and Jayanth G. Vedre MD, MPH

Objective: To compare the catheter-associated urinary tract infections (CAUTI) standardized infection rate (SIR) before and after implementation of a multimodal intervention approach in a rural tertiary hospital.

Design: Before-after analysis of a multimodal intervention to evaluate primary outcomes of the incidence of inpatient CAUTI, the SIR for CAUTI, and number of urinary catheter days.

Setting: All inpatient departments of a rural 504-bed tertiary care facility in the Midwest.

Participants: Patients admitted for in-patient care.

Interventions: A multimodal intervention composed of: (a) physician and nurse education, (b) modification of progress note templates and daily provider reminders for the clinical necessity of catheters, (c) implementing established best practices for eliminating CAUTIs, (d) advocating for alternative toileting options, and (e) promoting aseptic techniques for insertion and removal of catheters. SIR, CAUTI, and the number of urinary catheter days were obtained before and after implementation of the multimodal intervention in 2015 and 2017, respectively.

Results: After a one-year timeframe of intervention, CAUTI event rates decreased, and SIR for CAUTI was reduced by $60.2 \%$ (from I.524 to 0.607 ) with $P$ value $<0.05$. Urinary catheter days were also reduced from 16,195 in 2015 to 13,348 in 2017 (17.6\% reduction) with $P$ value $<0.05$.

Conclusions: Incidence of CAUTIs were significantly reduced with a team effort involving infection control, physician and nursing education, modification of progress note and templates and daily provider reminders for the clinical necessity of catheters and appropriate usage of urinary catheter with corresponding reduction in urinary catheter days.

Keywords: Catheter-associated urinary tract infections; Infection control;

Multimodal intervention; Standardized infection rate

Corresponding Author: Peter E. Umukoro, MD, MPH, ScD, Marshfield Clinic, Department of Internal Medicine, Marshfield, WI 54449, Tel: (617) 874-6908, Fax: (7I5) 387-5434, Email: peu258@mail.harvard.edu

Financial Support: The study was supported by the infection control team of the Marshfield Medical Center, Marshfield.
Received: November 8, 2019

Revised: March 20, 2020

Accepted: April 9, 2020

doi: $10.3121 / \mathrm{cmr} \cdot 2020.1533$

Disclosure: The work described in this manuscript was previously presented at Marshfield Medical Center Medical Education Day 
$\mathrm{C}$ atheter-associated (CA) urinary tract infection (UTI) is the fourth most common hospital-acquired infection ${ }^{1}$ and continues to be a threat to patient safety. In 2012, the incidence of CAUTI in the U.S.A. was 1.4 to 1.7 per 1,000 catheter days in inpatient adult and pediatric medical/ surgical floors per Centers for Disease Control and Prevention (CDC)/National Healthcare Safety Network (NHSN). ${ }^{2}$ Up to $69 \%$ of CAUTIs are preventable by implementing infection prevention practices. ${ }^{1}$ The CDC defines CAUTI with one or more of the following criteria: ${ }^{3}$ (1) urinary tract infection with an indwelling urinary catheter in place for $>2$ days on the date of event, with day of device placement being Day 1 and an indwelling urinary catheter in place on date of the event or the day before; (2) presence of at least one of the following signs or symptoms: fever $\left(>38.0^{\circ} \mathrm{C}\right)$, suprapubic tenderness, costovertebral angle pain or tenderness, urinary urgency or frequency, and dysuria; (3) urine culture with no more than two species of organisms and at least one culture $\geq 10^{5}$ colony forming units $(\mathrm{CFU}) / \mathrm{mL}{ }^{4}$

\section{According to Hooton et al: ${ }^{5}$}

The incidence of bacteriuria associated with indwelling catheterization is $3-8 \%$ per day, and the duration of catheterization is the most important risk factor for the development of CA-bacteriuria. Other risk factors associated with CA-bacteriuria include not receiving systemic antimicrobial therapy, female sex, positive urethral meatal culture results, microbial colonization of the drainage bag, catheter insertion outside the operating room, catheter care violations, rapidly fatal underlying illness, older age, diabetes mellitus, and elevated serum creatinine at the time of catheterization.

Complications associated with CA-bacteriuria include bacteremia (1\%-4\%) and death with a mortality rate of $\sim 13 \%{ }^{4,6}$ The reported attributable cost of a CAUTI adjusted to US dollars varies by inpatient department and ranges from $\$ 1,764$ for non-intensive care unit (ICU) patients to $\$ 10,197$ for ICU patients on Medicare per CAUTI event. ${ }^{7}$ Decreasing CAUTI rates and catheter days is necessary for acute care hospitals to improve quality of care and ensure patient safety.

Rates of CAUTI and other hospital-acquired infections are becoming publicly available data to guide patients in selection of facilities they would want to patronize. Hence, there is a growing financial and economic need by hospital administrations to healthily compete in bringing down infection rates to the barest minimum. It appears that the decreasing trends in CAUTI since 2012 have begun to plateau since implementation of multi-level strategies to reduce them. ${ }^{4,8,9}$ However, caution must be exercised to ensure these preventive strategies are maintained to prevent the resurgence of epidemic levels of hospital-acquired infections and CAUTIs seen in the last decade.
Our overall goal was to ensure patient safety and improve quality of care by implementing a multimodal intervention in all inpatient departments of an acute care facility in a rural Midwestern community and comparing our incidence of CAUTI and our SIR before and after this multimodal intervention. The described quality improvement program is a combination of best-practice recommendations from multiple studies $^{8,10-13}$ and provider suggestions with modifications for patient demographics such as age and health status (ICU versus observational). A multimodal intervention approach may improve CAUTI rates in medical complexes of similar size and patient population.

\section{Materials and Methods}

This quality improvement project used a before-after design with pre-/post-evaluation of hospital adverse outcomes. The multimodal CAUTI reduction program was implemented in a rural 504-bed community academic hospital in 2016. To encourage appropriate catheter use and reduce the number of urinary catheter days in inpatients, a multimodal intervention was implemented at all inpatient departments of a rural tertiary care facility in the Midwest.

\section{Case Definition}

We used the same case definition for CAUTI as the CDC in 2012 at both pre- and post-intervention to have fair comparisons of CAUTI rates at both time points. Previous CAUTI rates in 2015 would be overestimates due to bacterial colonization and fungiuria based on the redefinition of CAUTI by the NHSN in 2015., ${ }^{4,14}$

\section{Intervention}

Our multimodal approach was a strategy informed by our multidisciplinary team comprising of physicians, nurses, laboratory staff, in-hospital transporters, infection control, and quality initiative/quality assurance teams as well as effective strategies described in the literature. ${ }^{10-12,15,16}$ We used the validated PDSA(Plan, Do, Study, and Act) quality improvement model to develop a multimodal intervention ${ }^{17-19}$ that was feasible for our center.

A multimodal intervention whose implementation consisted of provider education, daily reminders for patient assessment of clinical necessity for catheter use, establishment of best practices, advocating for alternative toileting options, and promotion of aseptic technique for catheter insertion and removal was implemented in all inpatient departments from 2016 to 2017. Standardized technical protocols implemented included aseptic techniques for insertion and removal of catheters, maintaining closed drainage system, prevention of backflow, sterile technique for collecting urine for culture, and minimal duration of catheter placement. ${ }^{5,20}$

Other specific measures for the CAUTI reduction program included: (a) physician and nurse education including distribution of pocket cards with appropriate and inappropriate 
indications for urinary catheterization; (b) modification of progress note templates to remind providers about catheters in place and daily patient evaluation of the need for Foley catheters; and (c) monthly monitoring of CAUTI incidence and surveillance of nursing staff for catheter care procedures. ${ }^{20,21}$ The established "Best Practices" emphasized for eliminating CAUTIs are as follows: $:^{11,16}$

1) Limiting catheter use. Pocket cards highlighting appropriate and inappropriate indications for urinary catheter insertion.

2) Get catheters out as soon as possible.

3) Only ordering urine cultures when clinically indicated. A change in smell or color of urine ("the urine smells funny and looks awful") are not indications to culture.

4) Not ordering urinalysis and urine culture together. Ordering first urinalysis and then based on the results, if clinically indicated, to order a culture. Emphasizing the concept of asymptomatic bacteriuria.

5) Avoiding "pan culturing" or "reflexive culturing of urine" in response to a fever.

6) Advocate for alternative toileting options (e.g., urinals, condom catheters, measurement of diaper weight, intermittent catheterization (straight catheterization if needed).

\section{Outcomes}

Primary outcomes for analysis included the SIR, which is calculated by dividing the number of observed CAUTIs by the number of predicted CAUTIs per CDC protocol. ${ }^{3}$ Our secondary outcome was urinary catheter days and predicted CAUTIs. Urinary catheter days are the number of days during which a patient had an indwelling catheter per year.

\section{Evaluation}

SIRs for CAUTI were calculated one year prior to (in 2015) and one year after (in 2017) implementation of the multimodal intervention. This was to allow for a washout period in 2016 before and after the intervention. Suspected CAUTI infections were reviewed by infection control/hospital CAUTI prevention committee to confirm correct case definition.

Table 1. Catheter days, predicted and actual CAUTI and SIR for 2015 (pre-intervention) and 2017 (post-intervention).

\begin{tabular}{lllll}
\hline Year & $\begin{array}{l}\text { Catheter } \\
\text { Days }^{*}\end{array}$ & $\begin{array}{l}\text { Predicted } \\
\text { CAUTI* }\end{array}$ & CAUTI* & SIR $^{*}$ \\
\hline 2015 & 16,195 & 17.717 & 27 & 1.524 \\
2017 & 13,348 & 14.821 & 9 & 0.607 \\
\hline
\end{tabular}

CAUTI, Catheter-associated urinary tract infections; SIR, Standardized Infection Ratio. ${ }^{*} U$ statistic has $P$ value $<0.05$.

\section{Statistical Analyses}

Observed CAUTIs, predicted CAUTIs, calculated SIRs, and urinary catheter days were compared between pre- and postintervention among units using the Mann-Whitney $U$ test with two-sided hypothesis testing at a threshold level of significance at 0.05 . The SIR is a summary measure used to track hospital acquired infections at a national, state, or local level over time. The SIR adjusts for various facility and/or patient-level factors that contribute to hospital-acquired infection risk within each facility. Hospitals report their absolute number of infections, and the SIR and predicted CAUTIs are reported state-wide. The predicted CAUTI is the expected number of CAUTIs based on the hospital size. The SIR is the observed number of CAUTI divided by the predicted number of CAUTIs.

\section{Results}

Prior to implementation of the multimodal intervention, there were 16,195 urinary catheter days, 27 cases of confirmed CAUTI with a corresponding number of predicted infections of 17.717, and estimated calculated SIR of 1.524. Over a period of one year post-implementation of preventive measures, primary and secondary outcome measures decreased in most units. In 2017, the number of CAUTIs was nine, urinary catheter days were 13,348 , the predicted infection number was 14.821, and the estimated SIR was 0.607. Striking differences were noted for SIR, which was reduced from 1.524 to 0.607 for a $60.2 \%$ reduction over a 2 -year period, while the CAUTI event rate was reduced from 27 in 2015 to 9 in 2017 , a $66.6 \%$ reduction post-intervention. A $17.6 \%$ reduction in urinary catheter days were also noted from 16,195 in 2015 to 13,348 in 2017 . These were all statistically significant reductions with $P<0.05$ (Table 1 ). When data from individual units were reviewed, the greatest reductions were seen in the medical and surgical intensive care units (Table 2).

\section{Discussion}

CAUTI poses a major burden to patient health and safety by increasing both morbidity and mortality. Per a recent national survey, the use of evidence-based and recommended practices has increased in US hospitals which has led to increased CAUTI prevention rates and decreased CAUTI rates of $32 \%{ }^{6}$ Several US hospitals have been implementing different approaches to stem the tide of hospital-acquired infections since $2012 .^{9}$

In a comparable Canadian study during the same time period 2015-2017, there was a reduction of CAUTI rates from $2.4 \%$ in a 6 -month pre-intervention to $0.7 \%$ over a 6 -month period post-intervention. This represents a $71 \%$ decrease in CAUTI rates. Their multimodal intervention included reduction of routine catheter placement and auditing placement, aseptic catheter placement when needed and a closed drainage system, appropriate maintenance of catheters when in place, and earliest possible catheter removal with appropriate catheter removal protocols. ${ }^{22}$ 
Table 2. Catheter days, predicted and actual CAUTI and SIR for 2015 (pre-intervention) and 2017 (post-intervention) by unit.

\begin{tabular}{|c|c|c|c|c|c|c|c|c|}
\hline \multirow[b]{2}{*}{ Unit } & \multicolumn{4}{|c|}{2015} & \multicolumn{4}{|c|}{2017} \\
\hline & $\begin{array}{l}\text { Catheter } \\
\text { Days }\end{array}$ & $\begin{array}{l}\text { Predicted } \\
\text { CAUTI }\end{array}$ & \# of CAUTI & SIR & $\begin{array}{l}\text { Catheter } \\
\text { Days }\end{array}$ & $\begin{array}{l}\text { Predicted } \\
\text { CAUTI }\end{array}$ & \# of CAUTI & SIR \\
\hline $3 \mathrm{~N}$ & 1361 & 1.293 & 1 & 0.774 & 799 & 0.759 & 0 & $\mathrm{~N} / \mathrm{A}$ \\
\hline $4 N$ & 2202 & 2.091 & 2 & 0.956 & 1724 & 1.637 & 0 & 0.000 \\
\hline $5 \mathrm{~N}$ & 2041 & 2.152 & 5 & 2.324 & 1806 & 1.904 & 2 & 1.050 \\
\hline $6 \mathrm{~N}$ & 1343 & 1.416 & 0 & 0.000 & 1013 & 1.068 & 0 & 0.000 \\
\hline $7 \mathrm{~N}$ & 2920 & 2.773 & 0 & 0.000 & 2393 & 2.273 & 2 & 0.9404 \\
\hline $8 \mathrm{~N}$ & 781 & 0.823 & 2 & N/A & 784 & 0.827 & 1 & $\mathrm{~N} / \mathrm{A}$ \\
\hline MICU & 2399 & 2.595 & 6 & 2.312 & 1819 & 1.968 & 1 & 0.508 \\
\hline PEDS & 117 & 0.077 & 0 & N/A & 107 & 0.071 & 0 & $\mathrm{~N} / \mathrm{A}$ \\
\hline Peds cabin & 19 & 0.028 & 0 & N/A & 17 & 0.025 & 0 & $\mathrm{~N} / \mathrm{A}$ \\
\hline PICU & 220 & 0.308 & 1 & N/A & 109 & 0.153 & 0 & $\mathrm{~N} / \mathrm{A}$ \\
\hline SICU & 2792 & 4.160 & 10 & 2.404 & 2777 & 4.137 & 3 & 0.725 \\
\hline
\end{tabular}

CAUTI, Catheter-associated urinary tract infections; SIR, Standardized Infection Ratio; MICU=Medical Intensive Care Unit; PEDS/Peds

Cabin= Pediatric floor units, PICU=Pediatric Intensive Care Unit; SICU=Surgical Intensive Care Unit; N/A=Not Applicable

Although there was only a modest reduction in our catheter days pre- and post-intervention, our SIR for CAUTI was markedly reduced. Reducing exposure will reduce the likelihood for an adverse outcome; however, in cases where the exposure has been reduced to the minimum, a multimodal approach will work by mitigating other intervention points besides exposure.

A limitation of this single-center study is identifying which intervention works best to prevent CAUTIs. For example, some studies have suggested diagnostic stewardship. Although, this was not the goal of this study, we suppose that bundling together multiple interventions may act in a synergistic or at the least additive manner to reduce CAUTIs. ${ }^{6,21}$ Our results, although from a single center, may be generalizable to other parts of the developed world with the resources to address high infection rates but may be limited to facilities with a team dedicated to reviewing cases of suspected CAUTI and identifying ways to reduce these infections.

To maintain low CAUTI rates, healthcare providers and staff will continue to provide ongoing active surveillance and review breakthrough cases to identify and address contributing factors to CAUTI in future quality improvements. Based on our results and experience, we consider a multimodal intervention composed of provider education, establishment of best practices for catheter use and removal, and active surveillance by a provider team necessary elements of a quality improvement program to improve and maintain CAUTI rates in the hospital setting.

\section{Acknowledgements}

The authors acknowledge the editing assistance provided by Emily Andreae, $\mathrm{PhD}$ and Marie Fleisner.

\section{References}

1. Saint S, Greene MT, Krein SL, et al. A Program to Prevent Catheter-Associated Urinary Tract Infection in Acute Care. N Engl J Med. 2016;374(22):2111-2119.

2. Dudeck MA, Weiner LM, Allen-Bridson K, et al. National Healthcare Safety Network (NHSN) report, data summary for 2012, Device-associated module. Am J Infect Control. 2013;41(12):1148-1166.

3. CDC. Device Associated Module on UTI. Urinary Tract Infection (Catheter-Associated Urinary Tract Infection [CAUTI] and Non-Catheter-Associated Urinary Tract Infection [UTI]) and Other Urinary System Infection [USI]) Events. 2019. Available at: https://www.cdc.gov/nhsn/PDFs/ pscManual/7pscCAUTIcurrent.pdf. Accessed October 3, 2019.

4. Advani SD, Lee RA, Schmitz M, Camins BC. Impact of Changes to the National Healthcare Safety Network (NHSN) Definition on Catheter-Associated Urinary Tract Infection (CAUTI) Rates in Intensive Care Units at an Academic Medical Center. Infect Control Hosp Epidemiol. 2017;38(5):621-623.

5. Hooton TM, Bradley SF, Cardenas DD, et al; Infectious Diseases Society of America. Diagnosis, prevention, and treatment of catheter-associated urinary tract infection in adults: 2009 International Clinical Practice Guidelines from the Infectious Diseases Society of America. Clin Infect Dis. 2010;50(5):625-663.

6. Letica-Kriegel AS, Salmasian H, Vawdrey DK, et al. Identifying the risk factors for catheter-associated urinary tract infections: a large cross-sectional study of six hospitals. BMJ Open. 2019;9(2):e022137. 
7. Hollenbeak CS, Schilling AL. The attributable cost of catheterassociated urinary tract infections in the United States: A systematic review. Am J Infect Control. 2018;46(7):751-757.

8. Burton DC, Edwards JR, Srinivasan A, Fridkin SK, Gould CV. Trends in catheter-associated urinary tract infections in adult intensive care units-United States, 1990-2007. Infect Control Hosp Epidemiol. 2011;32(8):748-756.

9. Saint S, Greene MT, Fowler KE, et al. What US hospitals are currently doing to prevent common device-associated infections: results from a national survey. BMJ Qual Saf. 2019;28(9):741-749.

10. Blodgett TJ. Reminder systems to reduce the duration of indwelling urinary catheters: a narrative review. Urol Nurs. 2009;29(5):369-378, quiz 379.

11. Mody L, Meddings J, Edson BS, et al. Enhancing Resident Safety by Preventing Healthcare-Associated Infection: A National Initiative to Reduce Catheter-Associated Urinary Tract Infections in Nursing Homes. Clin Infect Dis. 2015;61(1):86-94.

12. Tedja R, Wentink J, O'Horo JC, Thompson R, Sampathkumar P. Catheter-Associated Urinary Tract Infections in Intensive Care Unit Patients. Infect Control Hosp Epidemiol. 2015;36(11):1330-1334.

13. Willson M, Wilde M, Webb ML, et al. Nursing interventions to reduce the risk of catheter-associated urinary tract infection: part 2: staff education, monitoring, and care techniques. J Wound Ostomy Continence Nurs. 2009;36(2):137-154.

14. Advani SD, Lee RA, Long M, Schmitz M, Camins BC. The Impact of 2015 NHSN Catheter-associated Urinary Tract Infection (CAUTI) Definition Change on Central Lineassociated Bloodstream Infection (CLABSI) Rates and CLABSI Prevention Efforts at an Academic Medical Center. Infect Control Hosp Epidemiol. 2018;39(07):878-880.

15. Carlson AL, Munigala S, Russo AJ, et al. Inpatient Urine Cultures Are Frequently Performed Without Urinalysis or Microscopy: Findings From a Large Academic Medical Center. Infect Control Hosp Epidemiol. 2017;38(4):455-460.

16. Fakih MG, Gould CV, Trautner BW, et al. Beyond Infection: Device Utilization Ratio as a Performance Measure for Urinary Catheter Harm. Infect Control Hosp Epidemiol. 2016;37(3):327-333.

17. Donnelly P, Kirk P. Use the PDSA model for effective change management. Educ Prim Care. 2015;26(4):279-281.

18. Laverentz DM, Kumm S. Concept Evaluation Using the PDSA Cycle for Continuous Quality Improvement. Nurs Educ Perspect. 2017;38(5):288-290.

19. Taylor MJ, McNicholas C, Nicolay C, Darzi A, Bell D, Reed JE. Systematic review of the application of the plan-dostudy-act method to improve quality in healthcare. BMJ Qual Saf. 2014;23(4):290-298.

20. Huang SS. Catheter-Associated Urinary Tract Infections Turning the Tide. N Engl J Med. 2016;374(22):2168-2169.

21. Patel PK, Gupta A, Vaughn VM, Mann JD, Ameling JM, Meddings J. Review of Strategies to Reduce Central LineAssociated Bloodstream Infection (CLABSI) and CatheterAssociated Urinary Tract Infection (CAUTI) in Adult ICUs. J Hosp Med. 2018;13(2):105-116.

22. Rozario D. Reducing catheter-associated urinary tract infections using a multimodal approach — the NSQIP experience of Oakville Trafalgar Memorial Hospital. Can J Surg. 2018;61(4):E7-E9.

\section{Author Affiliations}

Bindu R. Potugari, MD;* Peter E. Umukoro, MD, MPH, ScD;* and Jayanth G. Vedre, MD, MPH†

+Department of Internal Medicine, Marshfield Medical Center, Marshfield, WI USA

†Critical Care Medicine, Marshfield Medical Center, Marshfield, WI USA 